\title{
Primary Versus Deferred Ureteroscopy for Management of Calculus Anuria in Children: Review Article
}

\author{
Mostafa Abdel Razik Ahmed, Atef Fathi Ali, Mostafa Mohamed Mostafa*, Mohammad Sayed Abdel-Kader \\ Department of Urology, Faculty of Medicine, South Valley University, Egypt \\ *Corresponding author: Mostafa Mohamed Mostafa, Mobile: (+20)01069432827, E-mail: mostafaalsoghier@yahoo.com
}

\begin{abstract}
Background: The incidence of urolithiasis in the pediatric population is on the rise. Urolithiasis in the pediatric age group, although occurring less in adults, causes considerable morbidity. The role of ureteroscopy (URS) in the management of calculus anuria could be primary or deferred. The deferred URS after initial ureteral stenting or percutaneous drainage is the standard one. However, the remarkable progress in the manufacturing of ureteroscopes and lithotripters favored the primary URS.

Objective: This study aimed to evaluate the results of primary versus deferred URS for management of obstructive calculus anuria (OCA) in children in previous literatures.

Methods: We have searched literature in the American National Center for Biotechnology Information (NCBI), PubMed, Google scholar, Egyptian bank of knowledge, and science direct.

Conclusion: Primary URS is a definitive stone management technique and an appropriate option that can reduce hospital stay, prevent multiple anesthesia, and alleviate the costs in children with OCA when performed by skilled endourologists. However the deferred URS is a favored procedure for management of calculus anuria in pediatric patients compared to primary URS.
\end{abstract}

Keywords: Calcular anuria, Deferred, Ureteroscopy, Pediatric.

\section{INTRODUCTION}

With the development of small diameter endoscopes, improvement in the techniques of endoscopic lithotripsy in children has gradually become a major technique for the treatment of urinary stones ${ }^{(1)}$. The risk of anuria is associated with electrolyte imbalance, especially hyperkalemia, which leads to cardiac dysrhythmia and sudden death. Therefore, an imperative and urgent intervention must be considered. However, before making a decision of intervention we have to consider the availability of endoscopic experience and the instruments (2).

This work aimed to evaluate the results of primary versus deferred URS for management of obstructive calculus anuria in children in previous literatures.

\section{Calculus anuria in children:}

- Pathophysiology:

Renal, urologic, endocrine, and metabolic disorders may lead to the development of crystallized material in the urinary system. Materials that produce stones in the urinary tract of children include the following:

- Calcium with phosphate or oxalate.

- Purine derivatives.

- Magnesium and ammonium phosphate (struvite).

- Cysteine.

- Combinations of the preceding items.
- Drugs or their metabolites (e.g. phenytoin, triamterene).

- Melamine-contaminated milk powder consumption.

- Etiology:

Renal stones occur as a result of the following 3 factors:

- Supersaturation of stone-forming compounds in urine

- Presence of chemical or physical stimuli in urine that promote stone formation

- Inadequate amount of compounds in urine that inhibit stone formation (eg, magnesium, citrate).

In children, hypercalciuria and a reduction in the gap between calcium phosphate upper limit of metastability and supersaturation are significant risk factors for stones (3). Other factors such as developmental abnormalities of the urinary tract, urinary obstruction, urinary stasis, and infection with urea-splitting microorganisms may also be important.

- Epidemiology:

Frequency of urolithiasis in children has not been studied in a systematic population based fashion. Institutional and case reports indicate regional variation. A significant increase in the number of children diagnosed with and treated for urolithiasis has occurred in the last decade ${ }^{(4)}$.

- Management of ureteric stone in pediatric patients:

This article is an open access article distributed under the terms and conditions of the Creative Commons Attribution (CC BY-SA) license (http://creativecommons.org/licenses/by/4.0/) 


\section{A-Conservative Treatment:}

When a stone is small and at the ureteropelvic or ureterovesical junction, it may pass spontaneously, a few days of observation for spontaneous passage may be indicated prior to more aggressive intervention ${ }^{(5)}$.

\section{B-Dietary Measures:}

The overall role of diet is to supply adequate quantities of material for growth and metabolism without a surplus of relatively insoluble material that requires urinary excretion ${ }^{(5)}$.

\section{C-Surgical interventions:}

American Urological Association (AUA) guidelines to be applied to the pediatric patient. They concluded that calculi $<3 \mathrm{~mm}$ would pass spontaneously whereas calculi larger than $4 \mathrm{~mm}$ would require surgical management. The management of ureteral stones in children is becoming more similar to that in adults. With the ever continuing advance of technology, stone management has evolved from an open surgical approach into techniques that are significantly less invasive ${ }^{(6)}$.

\section{- Endoscopic lithotripsy:}

Endoscopic lithotripsy in children has gradually become a major technique for the treatment of ureteral stones. Currently, calculi throughout the entire upper urinary tract in children can be treated endoscopically using semi-rigid or flexible ureteroscopes with proven effectiveness and safety $\left(7,{ }^{8}\right)$. The stone-free rate following ureteroscopic lithotripsy for ureteral stones has been reported in as high as $98.5-100 \%{ }^{(9)}$. Large stones (stone burden exceeding $10 \mathrm{~mm}$ ) have a slightly lower clearance rate following one procedure ${ }^{(\mathbf{1 0})}$.

The overall complication rates have shown to range from $0 \%$ to $5.2 \%^{(\boldsymbol{9}, 10)}$. Flexible instruments are useful for antegrade ureteroscopy, as well as achieving ureteral access in children with complex anatomy ${ }^{(7)}$.

\section{- Extracorporeal shock wave lithotripsy:}

Extracorporeal shock wave lithotripsy has been the treatment of choice for symptomatic upper urinary tract stones in adults since 1980s, when the first-generation machines, featuring spark-gap electrodes, were introduced. Initially reported in 1986 large series of extracorporeal shockwave lithotripsy (ESWL)in children demonstrated complications, safety and stone-free rates similar to those in adults ${ }^{(11)}$.

\section{Ureteroscopy in pediatric patients:}

\section{- History of the Procedure:}

Progression from cystoscopy to upper urinary tract endoscopy was natural, with pediatric cystoscopes being used as the first rigid rod-lens ureteroscopes. The fiberoptic-based rigid endoscopes were small enough (averaging 7F in diameter) for frequent placement in the distal ureter under direct vision. The application of flexible ureteroscopy was first reported by Marshall ${ }^{(12)}$.

\section{- Contraindications:}

In general, the major contraindications are related to untreated infections and uncorrected bleeding diathesis prior to therapeutic endoscopy.

- Laboratory Studies:

Useful pre-operative laboratory stories include:

- Coagulation factors: Prothrombin time, activated partial thromboplastin time, and platelet count.

Urinalysis and urine culture:

- Standard preoperative laboratory workup: CBC count, electrolyte levels, and serum creatinine and BUN determination.

- Imaging Studies :

Useful preoperative imaging studies depending on the clinical presentation may include the following: Renal ultrasonography, IVP (once ubiquitous, now increasingly being phased out), CT scan, non-contrast (i.e. stone protocol), IV contrast with delay (i.e. Hematuria, Mass protocol), and MRI.

\section{Therapeutic Ureteroscopy:}

- Management of Stone Disease:

Ureteroscopy is a safe and minimally invasive method of treating stone disease in the kidneys and ureter as shown below. It can be used either as primary therapy or as salvage therapy for residual stones following treatment with other modalities such as ESWL and/or percutaneous nephrolithotomy (PCNL). Compared with ESWL, ureteroscopic lithotripsy achieves a greater stone-free state ${ }^{(\mathbf{1 3})}$.

- Minor intraoperative URS complications

In general, the minor complication rate (Colic/pain, fever, false passage, hematuria, extravasation, UTI, pyelonephritis) associated with ureteropyeloscopy was decreased based on refined technique, experience of the operators, and prompt treatment or prevention of intra-operative problems ${ }^{(14)}$.

- Major intraoperative URS complications:

Major intraoperative problems associated with ureteroscopy include trauma to tissues leading to significant ureteral wall perforations, avulsions, or foreign body (e.g., stone) migration into the ureteral wall. The major complication rate has markedly decreased (now occurring in less than $1 \%$ of all ureteroscopic procedures) ${ }^{(15)}$.

3. Primary versus deferred URS in pediatric patients:

In deferred URS Patients admitted were allocated to urinary drainage through the insertion of a JJ stent first then a deferred URS was performed after clinical and laboratory value improvement was noted. The improvement of endoscopic skills, development of small caliber ureteroscopes, modern lithotripters, and the use of flexible ureteroscopy (F-URS) to avoid 
ureteral stone migration and retrograde intra-renal disintegration have allowed more successful and safer endoscopic removal of ureteral stones ${ }^{(16)}$.

According to the joint EAU/AUA Nephrolithiasis Guideline Panel 2007, stones of $5 \mathrm{~mm}$ have a $68 \%$ chance of passing spontaneously (95\% CI: $46 \%$ to $85 \%$ ), and stones between $5 \mathrm{~mm}$ and $10 \mathrm{~mm}$ have a $47 \%$ chance (95\% CI: $36 \%$ to $59 \%){ }^{(17)}$.

Deferred URS characteristic by lower operative time as the first step in the treatment of OCA is to drain the obstructed system to allow the return of renal functions before definitive stone management, direct definitive stone management may lead to increased complications especially in this high-risk group of patients ${ }^{(18)}$. According to the American/ European Urological Association ureteral stones guidelines, the overall ureteroscopic stone-clearance for ureteral stones is $90 \%{ }^{(\mathbf{1 7})}$.

Elgammal et al. ${ }^{(19)}$ reported that initial drainage ID is associated with more complications, whereas formal surgery in compensated children was associated with a good outcome and lower complications in a study on 54 children with OCA. They reported $66 \%$ complications for PCN and $50 \%$ for JJ, whereas the complications with direct open surgery were only 13\%. Similar bilateral emergency URS associated with more morbidities ${ }^{(20)}$.

There were two studies on children with OCA and ARF. In the first study, which was done on 93 children, more complications were reported with direct DSM (30/93 patients) without initial drainage ID ${ }^{(21)}$. In the second study, which was done on 24 infants, Kotb et al. (22) reported that ID was a good choice while direct definitive stone management DSM without ID can be successfully performed by a skilled endourologist with a low risk of acceptable complications. JJ and PCN were inserted into 14 and six patients, respectively. The complication rate was $12.5 \%$ (one case of leakage and two cases of fever). The postoperative leakage occurred after ureterolithotomy, which was not preceded by ID ${ }^{(22)}$.

Hollenbeck et al. (20) reported increased risk of morbidity with bilateral URS, although Kotb et al. (22) reported that initial urinary drainage has been a good choice in the treatment of OCA with ARF in a study done on 24 infants. However Elgammal et al. ${ }^{(23)}$ reported that the initial urinary drainage is associated with a high complication rate.

A retrospective study included 66 children aged less than 12 years, who were subdivided into two groups reported that group A, which included 42 children who had undergone primary ureteroscopy without prestenting, and group B, which included 24 children who had undergone ureteroscopy after ureteric stenting. Secondary ureteroscopy is preferable over primary ureteroscopy in pediatric populations because of a significantly lower need for ureteric dilation, shorter procedure time and better stone clearance ${ }^{(23)}$.

A previous studies reported that bilateral emergency same-session URS is an appropriate option that can reduce hospital stay, prevent multiple anesthesia, and alleviate the costs in adult patients with OCA and ARF when performed by skilled endourologists ${ }^{(24)}$.

\section{CONCLUSION}

Primary URS is a definitive stone management technique and an appropriate option that can reduce hospital stay, prevent multiple anesthesia, and alleviate the costs in children with OCA and ARF when performed by skilled endourologists. However, the deferred URS is a favored procedure for management of calculus anuria in pediatric patients compared to primary URS due to higher stone free rate, shorter procedure time and with lower allover complications rate.

Financial support and sponsorship: Nil. Conflict of interest: Nil.

\section{REFERENCES}

1. Galal E, El-Bab T, Abdelhamid A (2013): Outcome of ureteroscopy for treatment of pediatric ureteral stones. Journal of Pediatric Urology, 9 (4): 476-8.

2. Fry A, Farrington K (2006): Management of acute renal failure. Postgraduate Medical Journal, 82 (964): 106-16.

3. Bergsland $\mathrm{K}$, Coe $\mathrm{F}$, White $\mathrm{M}$ et al. (2012): Urine risk factors in children with calcium kidney stones and their siblings. Kidney International, 81 (11): 1140-8

4. Routh J, Graham D, Nelson C (2010): Epidemiological trends in pediatric urolithiasis at United States freestanding pediatric hospitals. J Urol., 184 (3): 1100-4.

5. Marra G, Taroni F, Berrettini A et al. (2019): Pediatric nephrolithiasis:a systematic approach from diagnosis to treatment. Journal of Nephrology, 32 (2): 199-210.

6. Van Savage J, Palanca L, Andersen R et al. (2000): Treatment of distal ureteral stones in children: similarities to the American Urological Association guidelines in adults. The Journal of Urology, 164 (3 Part 2): 1089-93.

7. Smaldone M, Cannon G, Wu H et al. (2007): Is ureteroscopy first line treatment for pediatric stone disease?. The Journal of Urology, 178 (5): 2128-31.

8. Tan A, Al-Omar M, Denstedt $J$ et al. (2005): Ureteroscopy for pediatric urolithiasis: An evolving firstline therapy. Urology, 65: 153-6.

9. Minevich E, DeFoor W, Reddy $P$ et al. (2005): Ureteroscopy is safe and effective in prepubertal children. The Journal of Urology, 174 (1): 276-9.

10. Kim S, Kolon T, Canter D et al. (2008): Pediatric flexible ureteroscopic lithotripsy: The children's hospital of Philadelphia experience. J Urol., 180: 2616-9.

11. Muslumanoglu A, Tefekli A, Altunrende F et al. (2006): Efficacy of extracorporeal shock wave lithotripsy for ureteric stones in children. International Urology and Nephrology, 38 (2): 225-9. 
12. Marshall V (1964): Fiber Optics in Urology. J Urol., 91:110-4.

13. Aboumarzouk O, Kata S, Keeley F et al. (2011): Extracorporeal shock wave lithotripsy (ESWL): versus ureteroscopic management for ureteric calculi. Cochrane Database Syst Rev., 12: 29-35.

14. Jiang $\mathrm{H}$, Wu Z, Ding $Q$ et al. (2007): Ureteroscopic treatment of ureteral calculi with holmium: YAG laser lithotripsy. J Endourol., 21 (2): 151-4.

15. Huynh M, Telfer S, Pautler S et al. (2017): Retained Digital Flexible Ureteroscopes. J Endourol Case Rep., 3 (1): 24-27.

16. Hou W, Wen J, Ji Z et al. (2014): Reflex anuria: an old concept with new evidence. International Urology and Nephrology, 46 (2): 323-8.

17. Preminger G, Tiselius H, Assimos D et al. (2007): guideline for the management of ureteral calculi. The Journal of Urology, 178 (6): 2418-34.

18. Türk C, Knoll T, Petrik A et al. (2011): Guidelines on urolithiasis. European Association of Urology, Pp: 1-78. https://uroweb.org/wp-content/uploads/EAU-Guidelineson-Urolithiasis-2021-V2-1.pdf
19. Elgammal M, Abdel-Kader M, Kurkar A et al. (2009): Management of calculus anuria in children: Experience of 54 cases. Journal of Pediatric Urology, 5 (6): 462-5.

20. Hollenbeck B, Schuster T, Faerber G et al. (2003): Safety and efficacy ofsame-session bilateral ureteroscopy. Journal of Endourology, 17 (10): 881-5.

21. Ziada A, Sarhan O, Habib E et al. (2011): Assessment of recoverability of kidney function in children with obstructive calcular anuria: multicenter study. Journal of Pediatric Urology, 7 (3): 252-6.

22. Kotb S, EISheemy M, Morsi H et al. (2013): Renal recoverability in infants with obstructive calcular anuria: is it better than in older children? Journal of Pediatric Urology, 9 (6): 1178-82.

23. Elgammal M, Safwat A, Elderwy A et al. (2014): Primary versus secondary ureteroscopy for pediatric ureteral stones. Journal of Pediatric Urology, 10 (6): 1193 8.

24. Arda E, Cakiroglu B (2018): Bilateral same-session flexible ureterorenoscopy for renaland/or ureteric stone disease treatment. Arab Journal of Urology, 16 (4): 441-5. 